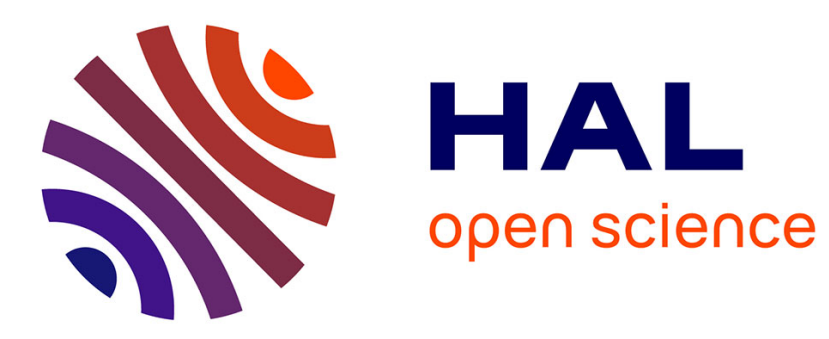

\title{
Variations on Hough-wavelet transforms for time-frequency chirp detection
}

\author{
Marcela Morvidone, Bruno Torrésani
}

\section{To cite this version:}

Marcela Morvidone, Bruno Torrésani. Variations on Hough-wavelet transforms for time-frequency chirp detection. Wavelets: Applications in Signal and Image Processing X, Sep 2003, San Diego, United States. 10.1117/12.505435 . hal-01300319

\section{HAL Id: hal-01300319 https://hal.science/hal-01300319}

Submitted on 10 Apr 2016

HAL is a multi-disciplinary open access archive for the deposit and dissemination of scientific research documents, whether they are published or not. The documents may come from teaching and research institutions in France or abroad, or from public or private research centers.
L'archive ouverte pluridisciplinaire HAL, est destinée au dépôt et à la diffusion de documents scientifiques de niveau recherche, publiés ou non, émanant des établissements d'enseignement et de recherche français ou étrangers, des laboratoires publics ou privés. 


\title{
Variations on Hough-wavelet transforms for time-frequency chirp detection
}

\author{
Marcela Morvidone ${ }^{a}$ and Bruno Torrésani ${ }^{b}$ \\ ${ }^{a}$ FYMA, Université Catholique de Louvain, Belgium. \\ ${ }^{b}$ LATP, Université de Provence, France.
}

\begin{abstract}
Several different approaches for joint detection/estimation of amplitude and frequency modulated signals embedded in stationary random noise with prescribed spectral density are considered and compared. Matched filter approaches are compared to time-frequency and time scale based approaches, together with "reassigned" versions. Particular attention is paid to the case of the so-called "power-law chirps", characterized by monomial and polynomial amplitude and frequency functions. As target application, the problem of gravitational waves at interferometric detectors is considered.
\end{abstract}

Keywords: detection/estimation, ambiguity function, wavelet transform, Hough transform, reassignment

\section{INTRODUCTION}

The detection of amplitude and frequency modulated signals embedded into stationary noise has become a fairly classical topic of signal decision theory. Besides the usual optimal filtering techniques, methods based upon time-frequency representations provide simple alternatives. Motivated by the recent development of large scale experiments for gravitational waves detection at interferometric detectors, we revisit the above mentioned problem and compare the performances of two different approaches, in the particular case of parametric classes of signals: power law chirps, embedded into stationary Gaussian noise, and some specific perturbed versions.

The first considered approach relies on an optimally matched filters bank, which may also be seen as a generalized cross-ambiguity function (GCAF.) Local maxima of the latter provide candidates for events. The corresponding statistical decision theory is fairly simple. However, this approach turns out to be quite fragile, which is a severe shortcoming in cases where the parametric model for the signal does not match perfectly the observation (which is the case for the particular application we consider, but also in many practical instances.)

The second approach is based on a (prewhitened) wavelet transform, followed by a Hough transform adapted to the candidate ridges in the time-scale plane. Again, local maxima of the Hough-wavelet transform (HWT) provide candidates for detected signals. However, as the HWT features much slower variations than the GCAF, the corresponding detector turns out to be more robust (at least within some region of the parameter space), and more suitable for coarse sampling, at the price of a decrease of the detection performances. We present the results of massive computer simulations which describe the behavior of the two approaches, as well as a possible alternative, using a reassigned wavelet transform followed by a Hough transform.

We describe the two approaches in some details, from theoretical as well as numerical point of views, and put them in competition in two situations. The first one corresponds to the case where the signal model used to build the detector matches perfectly the actual signal, and serves to analyze the performances of the two approaches in terms of detection. In the second situation, the signal model is slightly different from the actual signal, which serves as a test for the robustness of the proposed approaches.

Further author information: (Send correspondence to B.T.)

M.M.: E-mail: Marcela.Morvidone@fyma.ucl.ac.be, Telephone: +32 104732 36, FYMA, Université Catholique de Louvain, 2 chemin du cyclotron, B-1348 Louvain la Neuve, Belgium.

B.T.: E-mail: Bruno.Torresani@cmi.univ-mrs.fr, Telephone: +33 (4)91 0546 78, Address: LATP, Université de Provence, 39 rue Joliot-Curie, 13453 Marseille Cedex 13, France 
As a result, we show that (as expected) a finely sampled GCAF is certainly the best possible choice when possible. However, it suffers from a significant lack of robustness, it may lead to inacceptable computational burden for parametric families with larger parameter space. Among approximate methods, coarsely sampled GCAF and HWT appear as valuable compromises, each method outperforming the other one within some region of the parameter space. We also investigate a modification of the HWT, based upon a nonlinear wavelet transform: the "reassigned wavelet transform", obtained from the wavelet transform via a non-linear sharpening procedure, which enforces time-frequency localization. We show that even though the reassignment seems to perform satisfactorily, the corresponding RHWT (reassigned HWT) yields biased estimates for the parameters.

\section{CHIRP SIGNALS AND CORRESPONDING AMBIGUITY FUNCTIONS}

\subsection{Problem statement}

The joint detection-estimation problem may be formulated as follows: given a parametric family of signals $\left\{x_{\theta}, \theta \in \Theta\right\}$ labelled by a multidimensional parameter $\theta \in \Theta$, consider observations of the form

$$
\begin{aligned}
& H_{0}: \quad y(t)=X_{t}, \\
& H_{1}: \quad y(t)=A x_{\theta}(t)+X_{t},
\end{aligned}
$$

where $A \in \mathbb{R}^{+}$is an (unknown) multiplicative constant, and $X$ is a noise, modeled as a gaussian random process. The considered problem involves simultaneously hypothesis testing and parameter estimation under $H_{1}$. We assume that the noise $X$ is a gaussian second order random process, second order continuous and stationary, ${ }^{17,18}$ with absolutely continuous spectral measure $d \nu_{X}$. Therefore $X$ is completely characterized by its spectral density $\mathcal{S}_{X}$, and the Wiener-Khinchin theorem states that

$$
C_{X}(t, s):=\mathbb{E}\left\{X_{t} \bar{X}_{s}\right\}=C_{X}(t-s)=\int_{-\infty}^{\infty} e^{2 i \pi \nu(t-s)} \mathcal{S}_{X}(\nu) d \nu .
$$

Let $\mathcal{C}_{X}$ denote the corresponding covariance operator, defined by $\left\langle\mathcal{C}_{X} f, g\right\rangle=\mathbb{E}\{\langle X, g\rangle\langle f, X\rangle\}$ for all $f, g \in L^{2}(\mathbb{R})$ for which such an expression makes sense, we introduce

$$
\mathcal{E}_{X}=\left\{x \in L^{2}(\mathbb{R}), \mathcal{C}_{X}^{-1 / 2} x \in L^{2}(\mathbb{R})\right\} .
$$

A traditional approach typically makes use of the so-called generalized cross-ambiguity function (GCAF.)

Definition 1. Let $\left\{x_{\theta} \in \mathcal{E}_{X}, \theta \in \Theta\right\}$ be a parametric family of signals, embedded in zero mean, second order continuous and stationary random noise with given spectral density $\mathcal{S}_{X}$. Given $f \in \mathcal{E}_{X}$, the corresponding generalized cross ambiguity function* $\mathcal{A}_{f}^{(n)}$ is defined by

$$
\theta \rightarrow \mathcal{A}_{f}^{(n)}(\theta)=\frac{\left\langle f, \mathcal{C}_{X}^{-1} x_{\theta}\right\rangle}{\sqrt{\left\langle x_{\theta}, \mathcal{C}_{X}^{-1} x_{\theta}\right\rangle}}
$$

Keeping in mind the signal model (1) we have, under $H_{1}$

$$
\mathcal{A}_{y}^{(n)}(\theta)=A \mathcal{A}_{x_{\theta_{0}}}^{(n)}(\theta)+N(\theta) .
$$

The first term is the output signal and the second is the output noise. Considering the "signal" term, the following statement is a direct consequence of the Schwarz inequality.

Proposition 1. Under the assumptions of Definition 1, for $\theta_{0} \in \Theta$ and for all $\theta \in \Theta$ such that $x_{\theta}$ and $x_{\theta_{0}}$ are linearly independent,

$$
\left|\mathcal{A}_{x_{\theta_{0}}}^{(n)}(\theta)\right| \leq \mathcal{A}_{x_{\theta_{0}}}^{(n)}\left(\theta_{0}\right),
$$

\footnotetext{
*the superscript " $(n)$ " stands for "normalized".
} 
with equality if and only if $\theta=\theta_{0}$.

The output noise $N$ is a gaussian random function of $\theta$. Clearly, $\mathbb{E}\{N(\theta)\}=0$, and $\operatorname{Var}(N(\theta))=1$. Hence, the optimal $S N R^{\dagger}$ is given by

$$
\rho=\frac{\mathcal{A}_{x_{\theta_{0}}}^{(n)}\left(\theta_{0}\right)}{\sqrt{\operatorname{Var}(N(\theta))}}=\mathcal{A}_{x_{\theta_{0}}}^{(n)}\left(\theta_{0}\right)=\left\|\mathcal{C}_{X}^{-1 / 2} x_{\theta_{0}}\right\|
$$

This yields usual detection-estimation algorithms: given an observed signal $y$,

$i$. Compute the GCAF $\mathcal{A}_{y}^{(n)}(\theta)$ for a (suitably sampled) set of values for the parameters $\theta$.

ii. The values of $\theta$ such that $\mathcal{A}_{y}^{(n)}(\theta)$ exceeds a fixed threshold are marked as possible "events".

The amplitude parameter $A$ in (1) is also to be estimated from the observations. Clearly, once a signal with parameters $\theta_{0}$ has been detected, a natural estimate for $A$ is given by $\hat{A}=\mathcal{A}_{y}^{(n)}\left(\theta_{0}\right) / \rho$. However, too small values of $A$ make detection impossible. The detectability threshold, i.e. the smallest $A$ such that detection is considered possible, depends on the optimal SNR $\rho$.

Clearly enough, the "speed" of variation of the ambiguity function plays a key role in the detection problem. Rapidly varying functions $\mathcal{A}^{(n)}$ require a dense sampling of the parameter set, and thus yields high computational costs. Conversely, an unsufficiently dense sampling of the parameter set increases the risk of "missing the maximal value" of $\mathcal{A}^{(n)}$, and lowers the performances of the algorithm. The threshold controls the false alarm and missed detection rates. These rates will be used to evaluate and compare the performances of the different approaches. ${ }^{28}$

\subsection{Chirps, power law chirps, and their ambiguity functions}

A chirp is essentially a frequency and amplitude modulated signal, of the form

$$
x(t)=a(t) \cos (\varphi(t)),
$$

and additional assumptions on the relative speed of variations of the local amplitude $a$ and frequency $\varphi^{\prime}$ are generally made to ensure that the latter quantities have physical meaning. The instantaneous amplitude and frequency of $x \in L^{2}(\mathbb{R})$ are defined as follows. Consider the analytic signal $z_{x} \in L^{2}(\mathbb{R})$ and the corresponding canonical pair $\left(A_{x}, \phi_{x}\right)$ defined by

$$
z_{x}(t)=2 \int_{0}^{\infty} e^{2 i \pi \nu t} \hat{x}(\nu) d \nu, \quad A_{x}=\left|z_{x}\right|, \quad \phi_{x}=\arg \left(z_{x}\right) .
$$

$A_{x}, \phi_{x}$ and $\phi_{x}^{\prime}$ are respectively the instantaneous amplitude, instantaneous phase and the instantaneous frequency. Notice that the notions of local amplitude $a$ and frequency $\varphi$ which appear in (5) and instantaneous amplitude and frequency do not coincide in general ${ }^{11,25}$ (in such situations, the physical meaning of such quantities may however become questionable.) Nevertheless, under some additional assumptions, they may be shown to be fairly close to each other, as expressed by the following result. ${ }^{21,24}$

Proposition 2. Let $x$ be a chirp signal as in (5), such that $a \in C^{1}$ has bounded support and vanishes at boundaries, and $\varphi \in C^{2}$. Assume further that the local frequency is bounded from below: $\varphi^{\prime}>K$ for some $K \in \mathbb{R}^{+}$. Then

$$
z_{x}(t)=a(t) e^{i \varphi(t)}+r(t)
$$

where

$$
\|r\|_{\infty} \leq\|a\|_{1}\left(\frac{\left\|a^{\prime}\right\|_{1}}{\|a\|_{1}}\left\|\frac{1}{\varphi^{\prime}}\right\|_{\infty}+\left\|\frac{\varphi^{\prime \prime}}{\varphi^{\prime 2}}\right\|_{\infty}\right) .
$$

In other words, if the local amplitude and frequency vary slowly enough on the support of the signal, the analytic signal is close to the "exponential model" $a \exp (i \varphi)$. Results in the same spirit may also be obtained in the case of signals with infinite support, using a partition of unity.

\footnotetext{
†SNR stands for "Signal to Noise Ratio".
} 

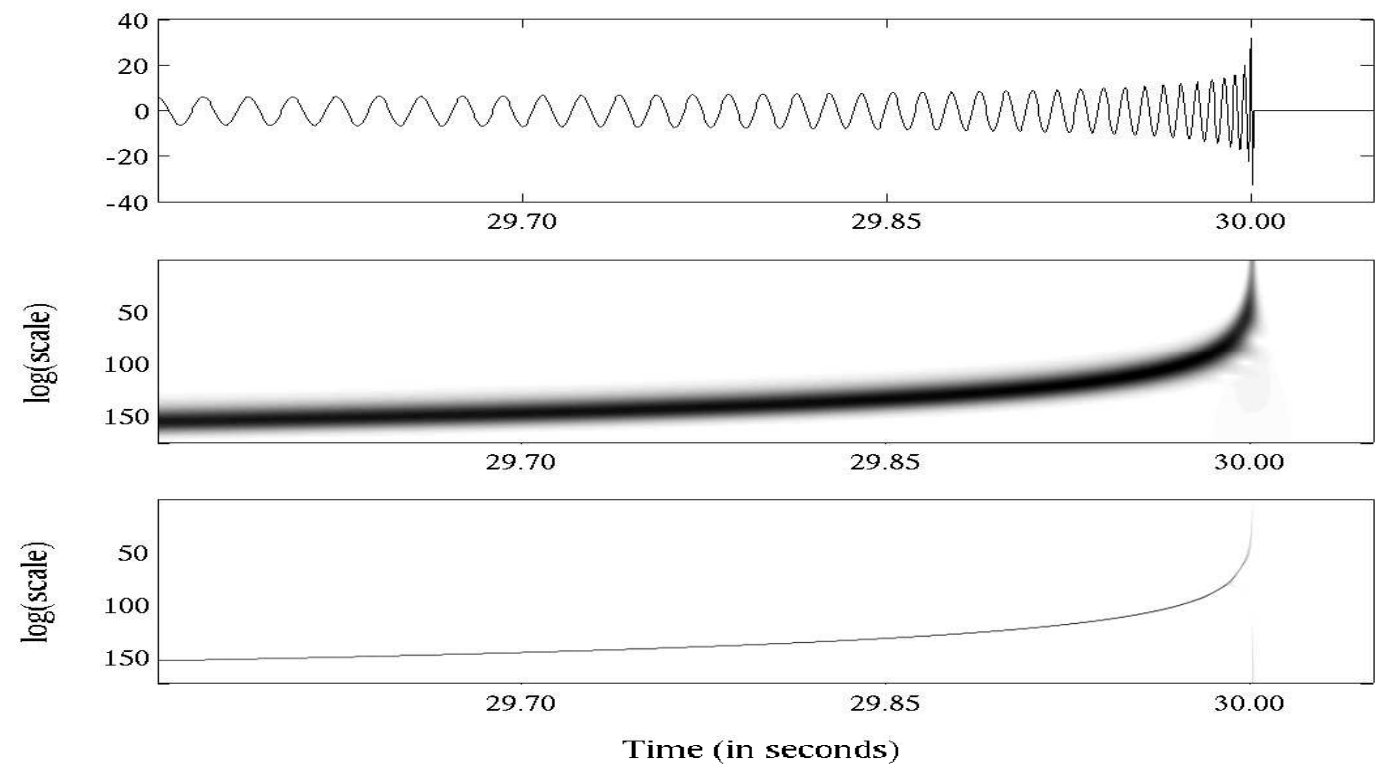

Figure 1. Example of power law chirp signal (top), together with its CWT square modulus (middle), and its reassigned wavelet transform (bottom.)

The Fourier representation of such signals is also of interest. The stationary phase approximations ${ }^{13}$ provide a way to estimate such Fourier transforms. Such approximations turn out to be extremely precise for very oscillatory signals, as shown by the following result. ${ }^{21,24}$

Proposition 3. Let $x=a \exp (i \varphi)$, where $a \in C^{2}$ is a positive-valued function, and $\varphi \in C^{5}$ is such that $\varphi^{\prime}>K>0$ for some constant $K$. Assume further that $\varphi^{\prime}$ is monotonic. Then, for all $\nu \in \operatorname{range}\left(\varphi^{\prime}\right) / 2 \pi$, we have

$$
\hat{x}(\nu)=\sqrt{2 \pi} \frac{a\left(t_{s}\right)}{\sqrt{\left|\varphi^{\prime \prime}\left(t_{s}\right)\right|}} e^{i\left(\varphi\left(t_{s}\right)-2 \pi \nu t_{s}\right)} e^{i \operatorname{sgn}\left(\varphi^{\prime \prime}\left(t_{s}\right)\right) \pi / 4}+r(\nu),
$$

where $t_{s}=t_{s}(\nu)$ is the (unique) solution of $\varphi^{\prime}\left(t_{s}\right)=2 \pi \nu$. The remainder $r$ depends upon the derivatives of $a$ and $\varphi$ up to degrees two and three respectively.

We shall focus on the class of so-called "power-law chirps", ${ }^{20}$ which have enjoyed a renewed interest lately because of the large scale gravity waves detection program. ${ }^{1,4}$ The latter are characterized by the power law behavior of their local amplitude and phase functions, of the form

$$
x(t)=\left(t_{0}-t\right)^{\alpha} \cos \left(\frac{2 \pi F_{0}}{\beta+1}\left(t_{0}-t\right)^{\beta+1}\right) H\left(t_{0}-t\right)
$$

( $t_{0}$ and $F_{0}$ being reference "time" and "frequency", and $H$ denoting Heaviside's step function.) A local frequency $\nu(t)=F_{0}\left(t_{0}-t\right)^{\beta}$ may be associated to such signals. $\alpha$ and $\beta$ are typically negative numbers, so that the local amplitude and frequency functions diverge as $t \rightarrow t_{0}$. Such models are supposed to reproduce (at first approximation order) the gravitational wave (that is, the wave of space-time metric deformation) generated by the coalescence of a binary system of two stars of masses $m_{1}$ and $m_{2}$. The constants $\alpha$ and $\beta$ are fixed by the physics of the problem (in that case, $\alpha=-1 / 4$ and $\beta=-3 / 8$ ), while the constant $F_{0}$ depends upon the masses of the two stars (essentially, the bigger the masses, the smaller $F_{0}$, even though the situation is a bit more complex.) An example of such power law chirp is shown in Figure 1.

An immediate consequence of Proposition 3 above is the approximate expression of the Fourier transform of such power law chirps. 
Corollary. Let $x$ be a power law chirp as in (10.) Then its Fourier transform is given by

$$
\hat{x}(\nu)=\hat{x}_{0}(\nu)+r(\nu)
$$

where the leading term reads

$$
\hat{x}_{0}(\nu)=\frac{1}{\sqrt{F_{0}|\beta|}}\left(\frac{\nu}{F_{0}}\right)^{(2 \alpha-\beta+1) / 2 \beta} \exp \left\{2 i \pi\left(-\nu t_{0}+F_{0}^{-1 / \beta} \frac{\beta}{\beta+1} \nu^{(\beta+1) / \beta}+\frac{1}{8}\right)\right\}
$$

and the remainder $r$ behaves as in Proposition 3 .

Such approximations are actually of quite questionable precision, at least for some ranges of values of $\nu$. The validity domain turns out to depend on the values of $\alpha$ and $\beta$. More precisely, ${ }^{21,24}$ for $\beta>-1$, such approximations are to be considered as low frequency approximations rather than high frequency ones. ${ }^{6,7}$ For $\beta \leq-1$, such chirps fall into the class of the infinitely oscillating functions studied in great details by Meyer and collaborators. ${ }^{20}$

The GCAF of power law chirps may be given fairly simple approximations. We shall take as signal model the Fourier representation provided by leading term of the stationary phase approximation. ${ }^{\ddagger}$ Let us then consider a reference signal with parameters $t_{0}$ and $F_{0}$ defined in the Fourier domain as in (12), copies with the same form and parameters denoted by $t$ and $F$, and study the corresponding GCAF. For the sake of simplicity (to avoid heavy notations and formulas), we shall consider the unnormalized version of the GCAF

$$
\mathcal{A}_{f}(\theta)=\left\langle f, \mathcal{C}_{X}^{-1} x_{\theta}\right\rangle
$$

rather than the normalized version $\mathcal{A}^{(n)}$ given in Definition 1 (the translation from one version to another is easily done.) It is easily seen that the generalized cross-ambiguity function $\mathcal{A}_{f}$ takes the form of a correlation between the input signal $f$ and a family of "templates" (in fact rescaled copies of a reference power law chirp $H(t)(-t)^{\alpha} \cos \left(2 \pi(-t)^{\beta+1}\right)$, modified by the action of $\mathcal{C}_{X}^{-1}$.) Hence, the detector based upon generalized cross ambiguity function may also be seen as a bank of matched filters, which are essentially rescaled copies of a reference signal, suitably filtered to correct for the spectral density of the underlying noise. By Plancherel's theorem, we have

$$
\mathcal{A}_{\left(t_{0}, F_{0}\right)}(t, F)=\int_{0}^{\infty} \overline{\hat{x}_{\left(t_{0}, F_{0}\right)}}(\nu) \hat{x}_{(t, F)}(\nu) \frac{d \nu}{\mathcal{S}_{X}(\nu)} .
$$

We introduce a few notations. We set

$$
\Delta t=t-t_{0}, \quad \Delta F=F^{-1 / \beta}-F_{0}^{-1 / \beta}, \quad M(\nu)=\frac{\nu^{(2 \alpha-\beta+1) / \beta}}{\mathcal{S}_{X}(\nu)},
$$

and we assume that $M \in L^{1}\left(\mathbb{R}^{+}\right)$(notice that such an assumption is essentially academic in practical situations, where the observed signal is automatically band-pass filtered by the signal acquisition device.) According to (12), the GCAF reads

$$
\begin{aligned}
\mathcal{A}_{\left(t_{0}, F_{0}\right)}(t, F) & \approx C\left(F_{0}, F\right) \mathcal{I}(\Delta t, \Delta F) \\
C\left(F_{0}, F\right) & =\frac{1}{|\beta|} \frac{1}{\left(F_{0} F\right)^{(2 \alpha+1) / 2 \beta}} \\
\mathcal{I}(\Delta t, \Delta F) & =\int_{0}^{\infty} M(\nu) \exp \left(2 i \pi\left\{\nu \Delta t-\frac{\beta}{\beta+1} \nu^{(\beta+1) / \beta} \Delta F\right\}\right) d \nu .
\end{aligned}
$$

Since $M \in L^{1}\left(\mathbb{R}^{+}\right)$, the latter integral is absolutely convergent, and $|\mathcal{I}(\Delta t, \Delta F)| \leq \mathcal{I}(0,0)$. Thus

$$
\left|\mathcal{A}_{\left(t_{0}, F_{0}\right)}(t, F)\right| \leq \frac{1}{|\beta|}\left(F_{0} F\right)^{-(2 \alpha+1) / 2 \beta}\|M\|_{1}
$$

\footnotetext{
${ }^{\ddagger}$ We limit ourselves to situations for which the frequency domain significantly involved in the ambiguity function is contained in the validity domain of the approximation.
} 

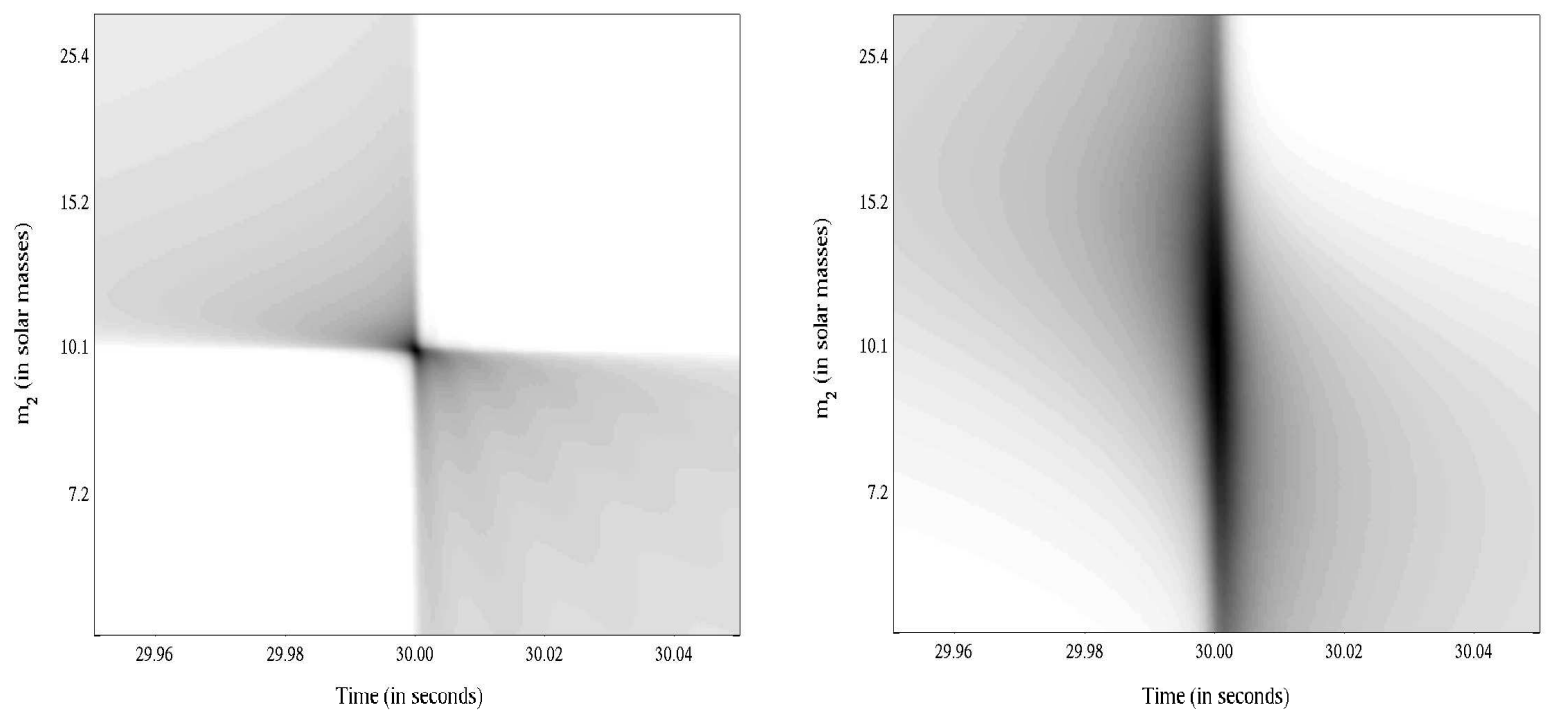

Figure 2. GCAF (left) and HWT (right) for a power-law chirps (corresponding to gravitational wave, generated by an inspiralling binary system, with masses $m_{1}=m_{2}=10 M_{\odot}$, in the Newtonian approximation)

with equality if and only if $\Delta F=\Delta t=0$, so that the GCAF can be used for detection purpose.

The integral $\mathcal{I}$ is again an oscillatory integral. In order to better understand its behavior, we use again a stationary phase argument. The order of magnitude of the integral depends upon the existence of stationary points of the argument of the integrated function, namely values of $t$ at which the latter argument vanishes. The following result ${ }^{21,24}$ gives first indications on the existence of such stationary points, and the corresponding behavior of the ambiguity function.

Theorem 1. With the above notations, assume $M \in L^{1}\left(\mathbb{R}^{+}\right)$. Assume further that $\Delta F \neq 0$ and $\Delta t \neq 0$. Then,

1. The integral in equation (17) admits a stationary point $\nu_{s}$ if and only if $\Delta t$ and $\Delta F$ have the same sign. $\nu_{s}$ is unique, and is given by $\nu_{s}=(\Delta t / \Delta F)^{\beta}$.

2. If $\Delta t$ and $\Delta F$ have the same sign, the leading term of the stationary phase approximation has modulus

$$
\left|\mathcal{I}_{0}(\Delta t, \Delta F)\right|=\sqrt{\frac{3}{8}}\left(\frac{\Delta F}{\Delta t}\right)^{\beta / 2} \frac{1}{\sqrt{|\Delta F|}} \frac{1}{\mathcal{S}_{X}\left(\left(\frac{|\Delta t|}{|\Delta F|}\right)^{\beta}\right)} .
$$

Proof. The first point results directly from the computation of the derivative of $f$, which vanishes if and only if $\Delta t=\nu^{1 / \beta} \Delta F$. For the second, we use the stationary phase lemma. ${ }^{13}$

Such an approximation turns out to be quite accurate, as may be seen on the left hand side image of figure 2 . The four quadrants appearing there correspond to the different signs for $\Delta F$ and $\Delta t$, i.e. to the existence or non-existence of stationary points. More complete numerical illustrations may be found in Refs. 21 and 24 .

\section{HOUGH-WAVELET TRANSFORM}

Since the signals under consideration (the chirp signals) possess specific properties of localization in timefrequency or time-scale spaces, it is natural to look for detection/estimation schemes that exploit these properties. ${ }^{5}$ Such an approach was already suggested in previous works. ${ }^{14,24}$ The trigonometric chirps possessing inherent scale covariance properties, the wavelet transform introduces itself naturally. 


\subsection{Wavelet transform and chirps}

We shall use a simple version of continuous wavelet transform (CWT.) More detailed accounts may be found in the literature. ${ }^{5,8,19}$ Let $\psi$ be an integrable and square-integrable function, such that $\hat{\psi}(\nu)=0$ for all $\nu \leq 0$, and that $0<c_{\psi}:=\int_{0}^{\infty}|\hat{\psi}(\nu)|^{2} \frac{d \nu}{\nu}<\infty$ Functions satisfying the above properties are called progressive wavelets. Wavelets provide alternative signal representations: let $x \in L^{2}(\mathbb{R})$ be a real-valued ${ }^{\S}$ function, and let $z_{x}$ denote its analytic signal as above. Then

$$
z_{x}=\frac{1}{c_{\psi}} \int T_{x}(b, s) \psi_{(b, s)} \frac{d s d b}{s^{2}}
$$

where the functions $\psi_{(b, s)}$ are the wavelets, shifted and rescaled copies of $\psi$ and the coefficients $T_{x}(b, s)$ form the Continuous wavelet transform $T_{x} \in L^{2}\left(\mathbb{R} \times \mathbb{R}^{+}, d b d s / s^{2}\right)$ of $x$.

$$
\begin{aligned}
\psi_{(b, s)}(t) & =\frac{1}{\sqrt{s}} \psi\left(\frac{t-b}{s}\right) \\
T_{x}(b, s) & =\left\langle x, \psi_{(b, s)}\right\rangle=\frac{1}{\sqrt{s}} \int_{-\infty}^{\infty} x(t) \bar{\psi}\left(\frac{t-b}{s}\right) d t
\end{aligned}
$$

The integral in (20) runs over the half plane $(b, s) \in \mathbb{R} \times \mathbb{R}^{+}$. Obviously, $x$ may be recovered as the real part of its analytic signal $z_{x}$, so that Equation (20) is enough to characterize it.

Random signals can be treated similarly. Given a second order continuous and stationary random process $X$ with spectral density $\mathcal{S}_{X} \in L^{\infty}$, set $\tilde{\psi}_{s}(t)=\frac{1}{\sqrt{s}} \bar{\psi}\left(-\frac{t}{s}\right)$. Then ${ }^{17,18}$ for any $s>0$, the process $T_{X}^{(s)}$ defined by the convolution $T_{X}^{(s)}=\tilde{\psi}_{s} * X$ is itself second order continuous and stationary, and has spectral density $\nu \rightarrow s|\hat{\psi}(s \nu)|^{2} \mathcal{S}_{X}(\nu)$. Hence, setting $T_{X}(b, s)=T_{X}^{(s)}(b)$, one may define the wavelet transform of the random signal $X$ as the family $T_{X}$ of random processes $\left\{T_{X}^{(s)}, s \in \mathbb{R}^{+}\right\}$. If $\psi$ has zero integral, then $\mathbb{E}\left\{T_{X}(b, s)\right\}=0$ for all $b, s$. In addition, one easily sees ${ }^{5}$ that $\mathbb{E}\left\{T_{X}(b, s) \overline{T_{X}}\left(b^{\prime}, s^{\prime}\right)\right\}=\left\langle\psi_{\left(b^{\prime}, s^{\prime}\right)}, \mathcal{C}_{X} \psi_{(b, s)}\right\rangle$. Finally, if the noise is assumed gaussian as before, the wavelet transform is also gaussian.

In what follows, considering the situation where a signal $x$ has been embedded into a random noise $X$ as above (with fixed spectral density $\mathcal{S}_{X}$ and covariance operator denoted by $\mathcal{C}_{X}$ ), we shall use a slightly different wavelet transform, the corresponding pre-whitened wavelet transform $\mathcal{W}_{x}$, defined as follows:

$$
\mathcal{W}_{x}(b, s)=\left\langle x, \mathcal{C}_{X}^{-1 / 2} \psi_{(b, s)}\right\rangle
$$

Clearly enough, the pre-whitened wavelet transform of the random noise $X$ reduces to a classical wavelet transform of a white noise. In particular, using the same notations as above, one has

$$
\begin{aligned}
\mathbb{E}\left\{\mathcal{W}_{X}(b, s)\right\} & =0, \\
\mathbb{E}\left\{\mathcal{W}_{X}(b, s) \overline{\mathcal{W}_{X}}\left(b^{\prime}, s^{\prime}\right)\right\} & =\left\langle\psi_{\left(b^{\prime}, s^{\prime}\right)}, \psi_{(b, s)}\right\rangle, \\
\operatorname{Var}\left\{\mathcal{W}_{X}(b, s)\right\} & =\|\psi\|^{2} .
\end{aligned}
$$

The purpose of this transform is to obtain a constant variance wavelet transform.

Let us return to the wavelet analysis of chirps. We essentially follow here refs 10 and 26 . Let us consider a model signal of the form (5) where the amplitude and phase functions are assumed to be twice and four times continuously differentiable respectively, and assume that the amplitude $a(t)$ is slowly varying compared to the oscillations. Let us consider a complex-valued (progressive) analyzing wavelet, which we write in the form $\psi(t)=a_{\psi}(t) e^{i \varphi_{\psi}(t)}$, and assume for the sake of simplicity that the (positive) amplitude $a_{\psi}$ is smooth and maximal at $t=0$, with $|\psi(0)|=1$, and that the wavelet is a linear phase one, i.e. is such that $\varphi_{\psi}^{\prime}(t)=\omega_{0}=2 \pi$. Then

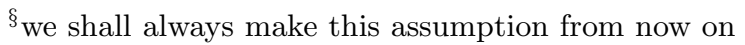


writing the continuous wavelet transform as an oscillatory integral, and approximating the integral by means of the stationary phase method, we obtain the following approximate expression ${ }^{10}$ for the wavelet transform of (5):

$$
T_{x}(b, s) \approx \sqrt{\frac{\pi}{2}} \frac{e^{i \pi \operatorname{sgn}\left(\Phi_{(b, s)}^{\prime \prime}\left(t_{s}\right)\right) / 4}}{\sqrt{s\left|\Phi_{(b, s)}^{\prime \prime}\left(t_{s}\right)\right|}} \bar{\psi}\left(\frac{t_{s}-b}{s}\right) z_{x}\left(t_{s}\right)
$$

where $\Phi_{(b, s)}(t)$ is the argument of the integrand, and the symbol $\operatorname{sgn}(t)$ stands for the $\operatorname{sign}$ of $t . t_{s}=t_{s}(b, s)$ is a stationary point of the integrand, i.e. a value of the argument such that $\Phi_{(b, s)}^{\prime}\left(t_{s}\right)=0$. In addition, it is assumed that for any $(b, s)$ under consideration, there exists one and only one such point, and that $\Phi_{(b, s)}^{\prime \prime}\left(t_{s}\right) \neq 0$ (otherwise, higher order approximations have to be used.)

Such stationary points have a very simple intuitive meaning: they represent the values of the integrand for which the oscillations of the wavelet match perfectly those of the signal. The precision of the approximation in (27) depends on the "speed of variations" of the oscillations of the integrand compared to its amplitude. However, the integrand involves both the signal and the scaled wavelet.

An alternative may be derived in the particular case of the so-called Morlet wavelet

$$
\psi(t)=e^{-t^{2} / 2 \sigma^{2}} e^{i \omega_{0} t},
$$

where the stationary phase argument may be refined to yield the following approximation

Proposition 4. Let $x$ be as in (5), and let $\psi$ be the Morlet wavelet. Then

$$
T_{x}(b, s)=\sqrt{\frac{\pi}{2}} \frac{e^{i \arctan \left(2 \sigma^{2} s^{2} \Phi_{(b, s)}^{\prime \prime}\left(t_{s}\right)\right) / 2}}{\sqrt{s \sqrt{\Phi_{(b, s)}^{\prime \prime}\left(t_{s}\right)^{2}+1 / \sigma^{4} s^{4}}}} \bar{\psi}\left(\frac{t_{s}-b}{s}\right) z_{x}\left(t_{s}\right)+r(b, s)
$$

where $r$ depends on derivatives of $a$ (resp. $\varphi$ ) of order up to two (resp. four.)

The proof of the proposition may be found in ref [24], together with more precise details on the remainder. Notice that in the limit $\sigma \rightarrow \infty$, one recovers the previous expression (27). It is also worth mentioning that the remainder is not uniform with respect to scale, which means that the precision of the approximation depends upon scale.

The approximate wavelet transforms in (27) and (29) are interesting in many respects. Let us simply point out that they naturally lead to the introduction of the following set of points

$$
\mathcal{R}=\left\{(b, s) \text { such that } t_{s}(b, s)=b\right\},
$$

termed the ridge of the wavelet transform. Indeed, if we forget for the sake of simplicity about the denominator in (27), we can see that $\left|T_{x}(b, s)\right|^{2}$ is locally maximum in the neighborhood of the ridge (this follows from the assumptions on the localization of the wavelet.) In addition, it is readily verified that the ridge takes the form of a curve in the time-scale plane

$$
s=s_{r}(b)=\frac{\varphi_{\psi}^{\prime}(0)}{\varphi^{\prime}(b)},
$$

where the argument $\varphi$ of the signal is defined in (5), and $\varphi_{\psi}$ is defined similarly. If the signal is locally monochromatic, the function $s_{r}(b)$ is monotonic and we may represent the ridge using the reciprocal function $b=b_{r}(s)$. Examples of such "ridge-localization" were given in several references. ${ }^{5,10,24}$ In the particular case of a power law chirp, as given in (5), the ridge takes the form of a curve in the $(b, s)$ half-plane, of equation

$$
b_{r}(s)=t_{0}-\left(\frac{2 \pi F_{0}}{\varphi_{\psi}^{\prime}(0)} s\right)^{-1 / \beta} \quad, \quad \text { or } \quad s_{r}(b)=F_{0}\left(t_{0}-b\right)^{\beta}, \quad s \in(0, \infty), \quad b \in\left(-\infty, t_{0}\right) .
$$

An exemple of such ridge localization may be found in figure 1, where the modulus of the CWT of a power law chirp signal is displayed in gray levels (middle image.)

\footnotetext{
ITaking advantage of the Gaussian amplitude.
} 


\subsection{The Hough-Wavelet transform}

The ridge localization may be utilized for detection purpose as follows. Notice first that since the wavelet $\psi$ is the Morlet wavelet, we have $\Phi_{(b, s)}^{\prime \prime}(t)=-\omega_{0} s_{r}^{\prime}(t) / s_{r}(t)^{2}$. Thus, it follows from equation $(27)$ that

$$
T_{x}\left(b, s_{r}(b)\right) \approx \sqrt{\frac{\pi}{2 \omega_{0}}} \sqrt{\left|\frac{s_{r}(b)}{s_{r}^{\prime}(b)}\right|} e^{-i \frac{\pi}{4} \operatorname{sgn}\left(s_{r}^{\prime}(b)\right) \overline{\psi(0)}} z_{x}(b)
$$

We show how to exploit such an expression for detection.

Remark : Notice also that according to the same approximations, we have

$$
\int\left|T_{x}\left(b_{r}(s), s\right)\right|^{2} \frac{d s}{s}=\int\left|\frac{s_{r}^{\prime}(b)}{s_{r}(b)}\right|\left|T_{x}\left(b_{r}(s), s\right)\right|^{2} d b=\frac{1}{2}\|x\|^{2}
$$

We are interested in detecting the presence of a frequency modulated signal from its wavelet transform. A now classical strategy ${ }^{5,11}$ consists in seeking the "optimal ridge" using Hough transform, i.e. the line in timescale plane which concentrates optimally the energy, within a family of "candidate ridges." Parametrizing ridges as functions of the scale variable $s \in\left[s_{0}, s_{1}\right] \rightarrow \rho(s)$, one looks for the "candidate ridge" $\rho$ which maximizes the line integral, ${ }^{24}$ or Hough transform

$$
\Gamma[\rho]=\int_{s_{0}}^{s_{1}}\left|T_{x}(\rho(s), s)\right|^{2} \frac{d s}{s} .
$$

Understanding precisely the behavior of the HWT is not straightforward. However, sufficient conditions may be obtained. $^{24}$ Introducing the auxiliary functions $\lambda: b \in\left[b_{0}, b_{1}\right] \rightarrow \lambda(b)=\rho\left(s_{r}(b)\right)$ and $\nu_{r}: b \rightarrow \nu_{r}(b)=\frac{\omega_{0}}{s_{r}(b)}$ (which may be interpreted as instantaneous frequency), it may be shown that if $\psi$ is sufficiently localized around the origin $t=0$, in the following sense

$$
\left|\psi\left(\frac{\nu_{r}(b)}{\omega_{0}}(b-\lambda(b))\right)\right|^{2}\left|\frac{\nu_{r}^{\prime}(b)}{\nu_{r}^{\prime}(\lambda(b))}\right| \leq|\psi(0)|^{2}, \quad \text { for all } b \in\left[b_{0}, b_{1}\right],
$$

then $\Gamma[\rho] \leq \Gamma\left[b_{r}\right]$ and the numerical maximization of $\Gamma$ will yield correct estimates for the seeked ridge.

In the situation at hand, the space of potential ridges is a finite dimensional space (a two-dimensional space in the case of power law chirps.) The ridge function takes the form

$$
s \rightarrow b_{t, F}(s)=t-\left(\frac{2 \pi F}{\omega_{0}} s\right)^{-1 / \beta}
$$

(recall that $\omega_{0}$ is a constant.) Thus, we are led to consider "energy" functions of the form

$$
\Gamma(t, F)=\Gamma\left[b_{t, F}\right]
$$

and seek maxima of such a two variables function with respect to $t$ and $F$.

Remark : According to the stationary phase approximation (27), given a candidate ridge $\rho$, we may write

$$
\left|T_{x}(\rho(s), s)\right| \approx C_{F_{0}}(s)\left|\psi\left(\left(\frac{\omega_{0}}{s^{\beta+1}}\right)^{1 / \beta} \Delta F-\frac{\Delta t}{s}\right)\right| .
$$

Therefore, if we still assume that $|\psi|$ is maximum at the origin, we see that this value may be attained only if $\Delta t$ and $\Delta F$ have the same sign. Under such a condition, one easily sees that the candidate ridge and the true ridge do intersect, which results in significantly large values for the line integral. This is reminiscent of the result of Proposition 3, and is confirmed by the numerical simulations, an example of which is displayed in Figure 2 (right image.) There, the $\Gamma$ functions shows a behavior quite similar to (a somewhat blurred version of) the ambiguity function $\mathcal{A}$, with a maximum at the same location. The behavior is also smoother, which suggests that more 
robust results could be obtained with that method. However, this only concerns "noise-free" situations, and the cases where the noise is present have to be studied more carefully.

The statistical behavior of the HWT is more difficult to understand. Under the $H_{0}$ hypothesis (noise only), the prewhitened wavelet transform of the signal $y$ is analogous to an ordinary wavelet transform of a gaussian white noise. Hence, all coefficients $\mathcal{W}_{y}(b, s)=\mathcal{W}_{X}(b, s)$ are also gaussian, and their square modulus $\left|\mathcal{W}_{y}(b, s)\right|^{2}$ follow a centered $\chi^{2}$ distribution. The line integral is therefore a sum of $\chi^{2}$ variables, but the latter being highly correlated, the distribution of the line integrals is very difficult to characterize precisely. The same holds true under the $H_{1}$ hypothesis: $\left|\mathcal{W}_{y}(b, s)\right|^{2}=\left|A \mathcal{W}_{x_{\theta}}(b, s)+\mathcal{W}_{X}(b, s)\right|^{2}$ is distributed according to an uncentered $\chi^{2}$ distribution (i.e. as a sum of squares of uncentered Gaussians), and the distribution of line integrals appears to be quite difficult to characterize (sum of correlated centered or non-centered $\chi^{2}$ variables.) Further work is clearly needed at this point, maybe following the lines developed by Hory and collaborators. ${ }^{15}$

\subsection{The "reassigned" version of Hough-wavelet transform"}

As we have stressed earlier, the HWT may be seen as a somewhat blurred version of the GCAF, due to the smoothing inherent to wavelet transformation. Recently, a family of techniques have been proposed which aim at (somewhat) restore the sharp localization properties of time-frequency or time-scale representations. The main point if to "deform" the representation by using time-frequency (or time-scale) vector fields (the "reassignment vector fields") and displace time-frequency (time-scale) coefficients accordingly. For a given reassignment prescription, it is possible to prove that in certain situations, such reassigned representations are indeed localized "where they should be"; however, this seems to be limited to a few specific situations, and the question of the practical value of such approaches is, in our opinion, still open. The reader interested in more details on such techniques is invited to refer to Ref. 7 for more details.

Nevertheless, it makes sense to test such practical applicability in the case study we devote this paper to. For this, we use a version of reassigned wavelet transform close to that used in Ref. 6 .

Let $\mathcal{R}: \mathbb{R} \times \mathbb{R}^{+} \rightarrow \mathbb{R} \times \mathbb{R}^{+}$be a map on the time-scale plane, called the reassignment map. Given $x \in L^{2}(\mathbb{R})$, consider its CWT $T_{x}$ and its spectrogram $\rho=\left|T_{x}\right|^{2} / c_{\psi} \in L^{1}\left(\mathbb{R} \times \mathbb{R}^{+}, d b d s / s^{2}\right)$, and associate with it the measure on $\mathbb{R} \times \mathbb{R}^{+}$defined as follows: for any $\Omega \subset \mathbb{R} \times \mathbb{R}^{+}$, denote by $\mathcal{R}^{-1} \Omega$ the inverse image of $\Omega$ by $\mathcal{R}$, i.e the set $\mathcal{R}^{-1} \Omega=\left\{(b, s) \in \mathbb{R} \times \mathbb{R}^{+}, \mathcal{R}(b, s) \in \Omega\right\}$. Then set

$$
M(\Omega)=\int_{\mathcal{R}^{-1} \Omega} \rho(b, s) \frac{d b d s}{s^{2}} .
$$

This defines a distribution in the time-scale half-plane, whose total mass equals the energy of the signal: $M(\mathbb{R} \times$ $\left.\mathbb{R}^{+}\right)=\|x\|^{2}$, and which may be used as an alternative to the scalogram. Notice that the practical implementation of such a transform from a CWT is quite easy, using a regular tiling of the time-scale half-plane and computing numerically $M\left(\omega_{k}\right)$ from the CWT for all tiles $\omega_{k}$. More details (in the spectrogram case) may be found in Ref 7 .

We used the following version of the reassignment map: writing the CWT as

$$
T_{x}(b, s)=\left|T_{x}(b, s)\right| e^{i \Phi(b, s)},
$$

we set

$$
\mathcal{R}(b, s)=(\tilde{b}(b, s), \tilde{b}(b, s))
$$

with

$$
\tilde{b}(b, s)=b+s^{2} \frac{\partial_{s} \Phi(b, s)}{\omega_{0}}, \quad \tilde{s}(b, s)=\frac{\omega_{0}}{\partial_{b} \Phi(b, s)}
$$

It is easily seen ${ }^{21}$ that such a prescription, which is quite close to the one studied in Ref 6 , provides optimal localization in the time-scale half-plane for specific classes of signals. Its application to power law chirps (see figure 1, bottom image) also shows a spectacular sharpening, compared to the classical scalogram (middle image.)

The corresponding line integral detection scheme RHWT (which stands for Reassigned Hough Wavelet Transform) is constructed along the lines of the HWT (applying the reassignment technique described above to the 


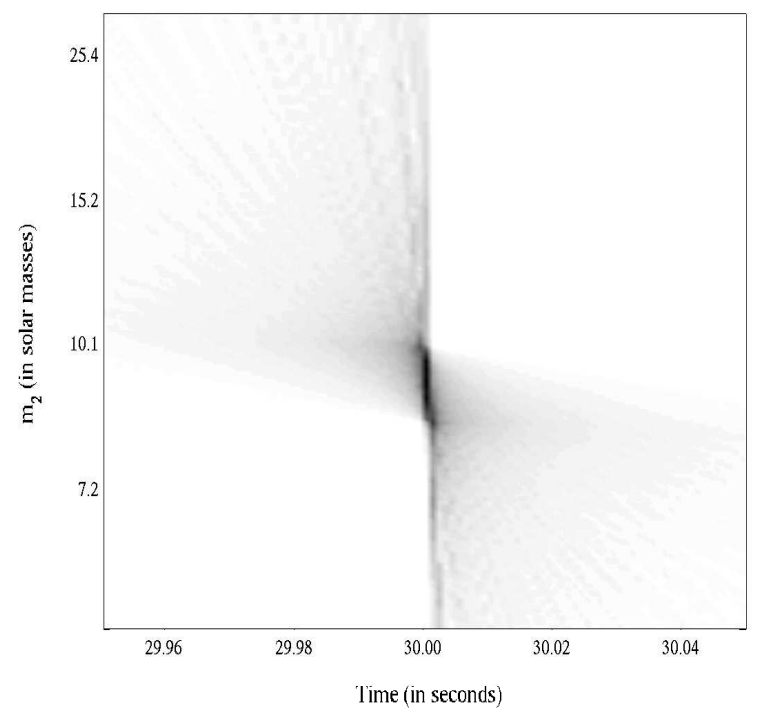

Figure 3. RHWT for a power-law chirp (corresponding to gravitational wave, generated by an inspiralling binary system, with masses $m_{1}=m_{2}=10 M_{\odot}$, in the Newtonian approximation)

"prewhitened" wavelet transform $\mathcal{W}_{x}$ instead of $T_{x}$.) As may be seen in figure 3 , the RHWT performs as expected, namely appears as a sharpened version of the usual HWT displayed in figure 2. However, it may also be seen that even though the localization is much sharper than before, the localization is not as precise, and the maximum in the "mass" space is not correctly located. This effect turns out to be quite systematic, and probably originates from the fact that the reassigned transform is not exactly located on the correct ridge, which induces a bias in the parameter estimation. For that reason, we shall focus below on the GCAF and the ordinary HWT for performance comparison. More details on the RHWT will be given in Ref 22 .

\section{COMPARISON BETWEEN THE TWO APPROACHES}

To evaluate and compare the performances of the two approaches, simulations were run for various values of the masses and distance parameters (hence various values of the SNR), and the two approaches were used. For the sake of simplicity, we will denote by $\mathcal{A}$ the detector based on GCAF, and by $\mathcal{R}$ the detector based upon HWT. The comparison strategy is as follows. For all configurations (masses and distance), $\mathcal{A}$ was used first, with several values for the detection threshold, and false alarm and missed detections were recorded for all values of the threshold. Two versions of $\mathcal{A}$ detectors were considered: a finely sampled one $\mathcal{A}_{f}$ (881 different values of $F$ ) and a coarser one $\mathcal{A}_{c}$ (160 different values of $F$.) $\mathcal{R}$ detector was also considered, with values of the threshold corresponding to the same false alarm rates as with the ambiguity function detector. The number of different values of $F$ was set to 160 (i.e. the same number as the coarsely sampled detector $\mathcal{A}_{c}$.) The results (i.e. the detection percentages, for fixed false alarm rates) are displayed in columns 4,5 and 6 of Table 1 . As expected, $\mathcal{A}_{f}$ outperforms both $\mathcal{A}_{c}$ and $\mathcal{R}$ in all situations. More interesting is the comparison of $\mathcal{A}_{c}$ and $\mathcal{R}$. The latter actually shows that the performances of $\mathcal{R}$ are better than those of $\mathcal{A}_{c}$ for small values of the masses, while the opposite result is obtained for larger masses. Therefore, HWT methods appears as a valuable alternative to the GCAF, within a given range of the parameter space.

However, an important issue is that of the robustness of the considered approaches with respect to model variations. In other words, what happens when the model on which the detectors are based is not strictly matched to the actual signal ? In the particular case of chirp signals, gravity waves models provide simple case studies for testing the robustness of the proposed methods. 


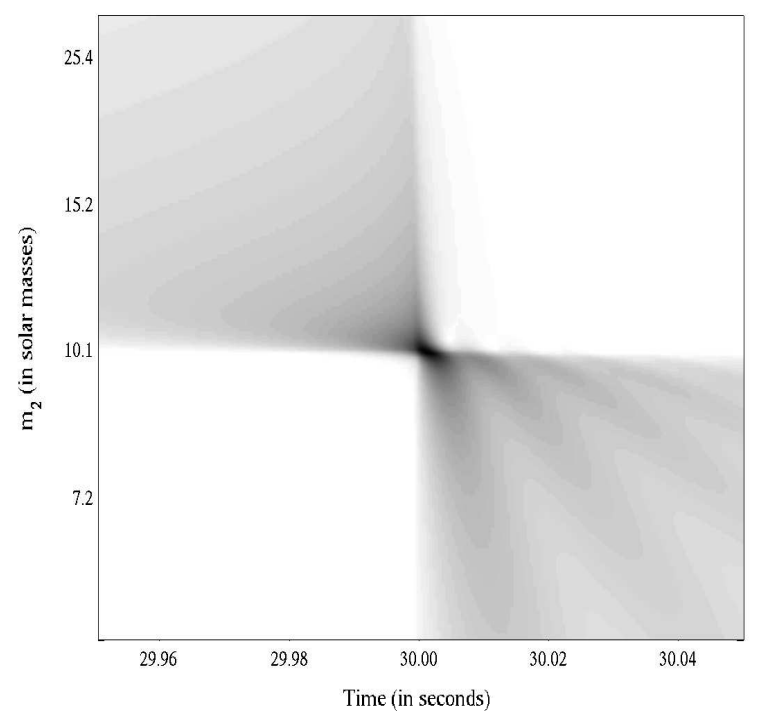

Figure 4. GCAF for a power-law chirp (corresponding to gravitational wave, generated by an inspiralling binary system, with masses $m_{1}=m_{2}=10 M_{\odot}$, in the post-Newtonian PN2 approximation)

We considered a signal model slightly different from the Newtonian approximation of gravity waves, provided by higher order post-Newtonian approximations $\|$. The signal model is here given by

$$
x(t)=\left(t_{0}-t\right)^{\alpha} \cos (g(t)) H\left(t_{0}-t\right),
$$

where the amplitude $\left(t_{0}-t\right)^{\alpha}$ is the same as in the Newtonian situation, and the argument reads ${ }^{2,3}$ :

$$
g(t)=\phi_{0}-\frac{2}{\eta}\left\{\Theta^{5 / 8}+\left(\frac{3517}{8064}+\frac{55}{96} \eta\right) \Theta^{3 / 8}-\frac{3 \pi}{4} \Theta^{1 / 4}+\left(\frac{9275495}{14450688}+\frac{284875}{258048} \eta+\frac{1855}{2048} \eta^{2}\right) \Theta^{1 / 8}\right\} .
$$

Here, $\Theta(t)=\frac{\eta}{5 T_{\odot} M}\left(t_{0}-t\right)$ and $\eta=\frac{\mu}{M}$, where $M=m_{1}+m_{2}$ and $\mu=m_{1} m_{2} /\left(m_{1}+m_{2}\right)$ are respectively the total mass and the reduced mass of the binary system. $\phi_{0}$ is a global phase shift. Contrary to the Newtonian approximation, in which the dependance with respect to masses was collected into a single constant $F_{0}$, the argument $g$ now depends on the masses $m_{1}$ and $m_{2}$ independently. The first term of the development actually coincides with the argument of the Newtonian model (10), while the other terms represent more complex corrections.

As may be seen on Figure 4, the global behavior of the GCAF is only weakly modified by the change of approximation order, when the signal model used for building the GCAF coincides with the observed signal (matched situation.) However, in the mismatched situations, significant deviations from the ideal situation are observed, as may be seen for example in Figure 5. On one hand, the peak value appears to be significantly smaller than in the matched case, ${ }^{21}$ which is a bad news for detection purpose. Also, the behavior near the maximum of the GCAF and the HWT is significantly modified, yielding a potentially incorrect estimation of parameters. Further numerical results ${ }^{21}$ definitely confirm this statement. Therefore, it makes sense to study and compare systematically the robustness of the two methods with respect to such deviations.

In order to asssert the robustness of the two approaches under consideration, we performed the same simulations as before, in the mismatched case. The last three columns of Table 1 provide numerical estimates for detection percentages, for fixed false alarm rates, and various values of the distance at which coalescence takes place. The results obtained here confirm the results discussed above: while optimal results are obtained with

\footnotetext{
${ }^{\|}$For the considered problem, such approximations are questionable as well, in particular in the last stage of the inspiral of the system.
} 

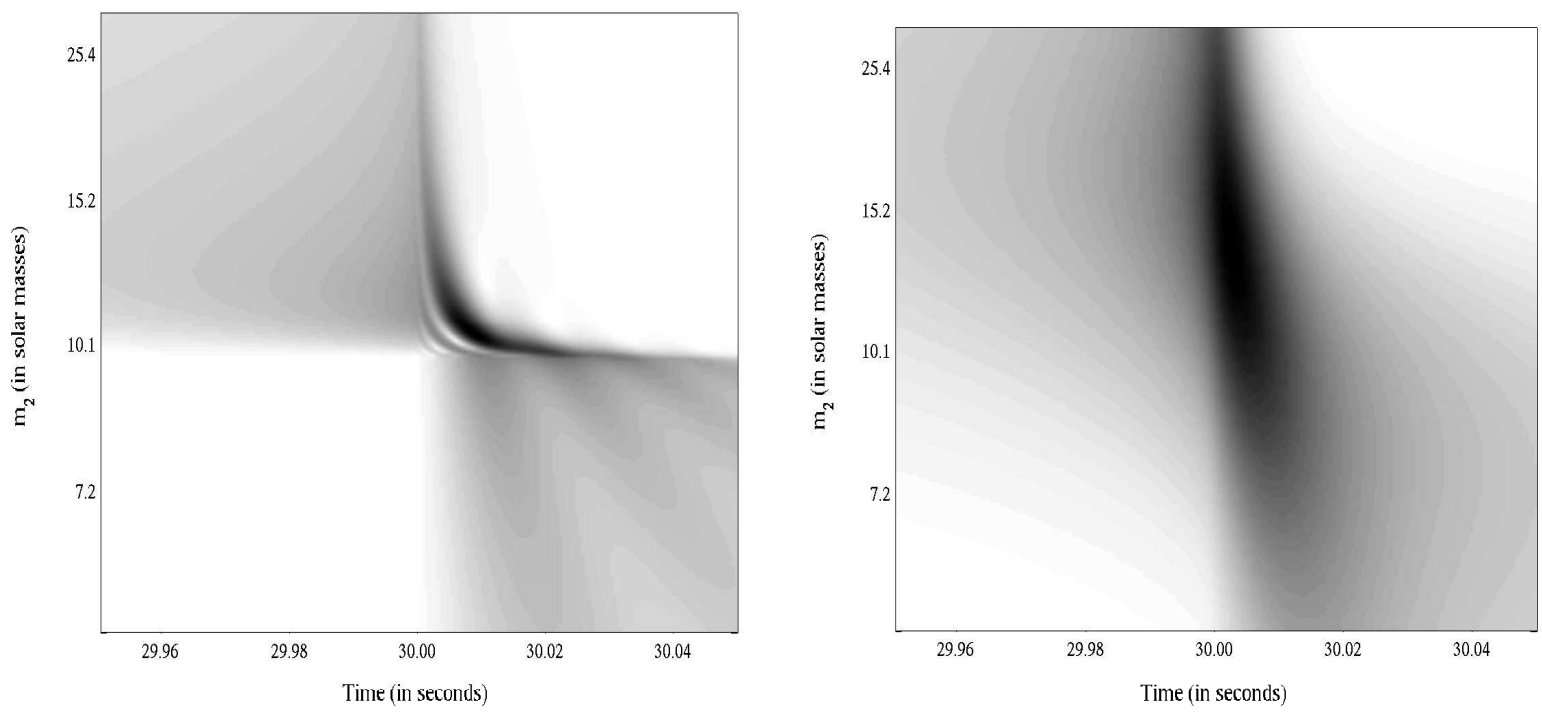

Figure 5. mismatched situation: GCAF (left) and HWT (right) for a power-law chirps (corresponding to gravitational wave, generated by an inspiralling binary system, with masses $m_{1}=m_{2}=10 M_{\odot}$, in the post-Newtonian PN2 approximation, analyzed with Newtonian detectors)

the finely sampled $\mathcal{A}_{f}$ detector, the $\mathcal{R}$ detector outperforms the coarsely GCAF detector $\mathcal{A}_{c}$ within the "small masses" region of the parameter space.

A more detailed analysis, including the discussion of ROC curves and the analysis of a variant algorithm utilizing reassigned wavelet transforms, will be published elsewhere. ${ }^{22}$

\section{CONCLUSIONS}

We have reported in this paper recent results on time-scale detection for power law frequency modulated signals. The main goal was to study systematically the strategies proposed by several authors, ${ }^{7,14}$ and compare them with the optimal approaches based upon banks of matched filters. More precisely, we have focused on the comparison of the Hough wavelet transform with the matched filters. The main results of our study are the following:

1. As expected the optimal approach outperforms the HWT approach, if "practical" issues (including computational burden, robustness,...) are not taken into account.

2. Taking into account computational issues, it turns out that subsampling may affect the behavior of the GCAF significantly. Depending on the values of the parameters, the HWT may happen to be more robust to subsampling than GCAF.

3. Similar conclusions may be drawn concerning the robustness with respect to errors in the model. The signals under consideration being extremely oscillatory, a small mismatch between the model and the observed signal may have dramatic consequences on detection. Again, within a given region of the parameter space, the HWT appears to be more robust with respect to model perturbations, at least for the type of perturbations that have been considered.

More complete results (in particular concerning the behavior of the GCAF and robustness issues) may be found in Ref. 21. This work may be expanded in several respects. More detailed results on detection/estimation will be published in Ref. 22, where a variant to the HWT, using the reassigned wavelet transform as suggested by Chassande-Mottin and Flandrin ${ }^{7}$ will be studied in more details. 
Table 1. Detection percentages for the $\mathcal{A}_{g}, \mathcal{A}_{f}$ and $\mathcal{R}$ detectors for different masses, distances and false alarm rates. Expected signal: inspiralling binaries gravity waves, in Newtonian approximation. Observed signals: inspiralling binaries gravity waves, in Newtonian approximation (matched case) and Post Newtonian approximations (mismatched case.)

\begin{tabular}{|c|c|c|c|c|c|c|c|c|}
\hline Masses & $\begin{array}{l}\text { Distance } \\
\text { and SNR }\end{array}$ & $\begin{array}{c}\text { False alarm } \\
\text { per sec. }\end{array}$ & $\begin{array}{c}\mathcal{R} \\
\text { (match) }\end{array}$ & $\begin{array}{c}\mathcal{A}_{c} \\
\text { (match) }\end{array}$ & $\begin{array}{c}\mathcal{A}_{f} \\
\text { (match) }\end{array}$ & $\begin{array}{c}\mathcal{R} \\
\text { (mismatch) }\end{array}$ & $\begin{array}{c}\mathcal{A}_{c} \\
\text { (mismatch) }\end{array}$ & $\begin{array}{c}\mathcal{A}_{f} \\
\text { (mismatch) }\end{array}$ \\
\hline \multirow{9}{*}{$\begin{array}{l}m_{1}=1.4 M_{\odot} \\
m_{2}=1.4 M_{\odot}\end{array}$} & & 1 & 100 & 98 & 100 & 100 & 60 & 100 \\
\hline & $10 \mathrm{Mpc}$ & 2 & 100 & 99 & 100 & 100 & 67 & 100 \\
\hline & $\rho=13.1$ & 3 & 100 & 99 & 100 & 100 & 73 & 100 \\
\hline & & 1 & 57 & 26 & 100 & 59 & 10 & 100 \\
\hline & $15 \mathrm{Mpc}$ & 2 & 67 & 29 & 100 & 63 & 15 & 100 \\
\hline & $\rho=8.74$ & 3 & 79 & 35 & 100 & 73 & 21 & 100 \\
\hline & & 1 & 12 & 6 & 96 & 20 & 3 & 100 \\
\hline & $20 \mathrm{Mpc}$ & 2 & 22 & 15 & 97 & 26 & 5 & 100 \\
\hline & $\rho=6.55$ & 3 & 34 & 16 & 98 & 33 & 7 & 100 \\
\hline \multirow{9}{*}{$\begin{array}{l}m_{1}=10 M_{\odot} \\
m_{2}=1.4 M_{\odot}\end{array}$} & & 1 & 70 & 97 & 100 & 32 & 34 & 55 \\
\hline & $45 \mathrm{Mpc}$ & 2 & 76 & 97 & 100 & 43 & 35 & 59 \\
\hline & $\rho=6.77$ & 3 & 81 & 98 & 100 & 52 & 39 & 64 \\
\hline & & 1 & 51 & 90 & 99 & 20 & 26 & 45 \\
\hline & $50 \mathrm{Mpc}$ & 2 & 62 & 93 & 99 & 27 & 32 & 49 \\
\hline & $\rho=6.10$ & 3 & 73 & 94 & 100 & 38 & 33 & 51 \\
\hline & & 1 & 24 & 58 & 96 & 9 & 11 & 12 \\
\hline & $60 \mathrm{Mpc}$ & 2 & 28 & 63 & 97 & 15 & 13 & 15 \\
\hline & $\rho=5.08$ & 3 & 34 & 65 & 98 & 22 & 15 & 20 \\
\hline \multirow{9}{*}{$\begin{array}{l}m_{1}=10 M_{\odot} \\
m_{2}=10 M_{\odot}\end{array}$} & & 1 & 83 & 99 & 99 & 46 & 58 & 84 \\
\hline & $145 \mathrm{Mpc}$ & 2 & 86 & 99 & 99 & 51 & 70 & 88 \\
\hline & $\rho=5.18$ & 3 & 87 & 99 & 99 & 62 & 75 & 88 \\
\hline & & 1 & 65 & 97 & 98 & 53 & 47 & 83 \\
\hline & $150 \mathrm{Mpc}$ & 2 & 73 & 99 & 99 & 56 & 53 & 86 \\
\hline & $\rho=5.00$ & 3 & 77 & 99 & 99 & 69 & 57 & 88 \\
\hline & & 1 & 60 & 97 & 97 & 41 & 45 & 71 \\
\hline & $160 \mathrm{Mpc}$ & 2 & 67 & 98 & 98 & 50 & 54 & 72 \\
\hline & $\rho=4.69$ & 3 & 69 & 99 & 99 & 56 & 56 & 76 \\
\hline
\end{tabular}

Concerning the target application under study, the conclusion of our analysis seems to be that strategies such as HWT do have a potential as a "trigger" algorithm for fast detection of potential events, which would be refined afterwards using (more costly) optimal approaches.

\section{ACKNOWLEDGMENTS}

We wish to thank J.M. Innocent and E. Chassande-Mottin for stimulating discussions.

Part of M. Morvidone's work was supported by a grant of the Fondo para el Mejoramiento de la Calidad Universitaria de la República Argentina. She is currently a Marie Curie fellow of the European commission, in the Research Training Network Harmonic Analysis and Statistics for Signal and Image Processing.

This paper was written while B. Torrésani was a host of Institut Henri Poincaré, Paris. 


\section{REFERENCES}

1. A. Abramovici et al., LIGO: the Laser Interferometer Gravitational-Wave Observatory, Science, Vol. 256, pp. 325-333 (1992).

2. L. Blanchet, T. Damour and B. Iyer, Gravitational waves from inspiralling compact binaries: Energy loss and waveform to second-post-Newtonian order, Physical Review, D51:10 (1995), pp. 5360-5386.

3. L. Blanchet, B. Iyer, C. Will and A. Wiseman, Gravitational waveforms from inspiralling compact binaries to second-post-Newtonian order, Class. Quantum Grav., 13 (1996), pp. 575-584.

4. C. Bradaschia et al. The Virgo project : a wide band antenna for gravitational wave detection, Nucl. Instrum. Methods Phys. Res. Vol. A289, pp. 518-525 (1990).

5. R. Carmona, W. Hwang and B. Torrésani, Practical Time-Frequency Analysis. Gabor and Wavelet Transforms with an Implementation in $S$ (Academic Press, 1998).

6. E. Chassande-Mottin, Méthodes de réallocation dans le plan temps-fréquence pour l'analyse et le traitement de signaux non-stationnaires, PhD Thesis (Université de Cergy-Pontoise, 1998).

7. E. Chassande-Mottin and P. Flandrin, On the time-frequency detection of chirps, (Appl. Comp. Harm. Anal., Vol. 6, pp. 252-281 (1998).

8. I. Daubechies, Ten Lectures on Wavelets CBMS-NFS Regional Series in Applied Mathematics 61, (SIAM, 1992).

9. L. Daudet, M. Morvidone and B. Torrésani, Time-frequency and time-scale vector fields for deforming timefrequency and time-scale representations, Proceedings of the SPIE conference, Denver, M. Unser Ed., pp. 2-15. (1999).

10. N. Delprat, B. Escudié, P. Guillemain, R. Kronland-Martinet, Ph. Tchamitchian and B. Torrésani, Asymptotic wavelet and Gabor analysis: extraction of instantaneous frequencies, IEEE Trans. Inf. Th., Vol. 38, pp. 644-664 (1992).

11. P. Flandrin, Temps-Fréquence, Traité des Nouvelles Technologies, série Traitement du Signal, (Hermès, 1993).

12. A. Grossmann, J. Morlet, Decomposition of Hardy functions into square integrable wavelets of constant shape, SIAM J. of Math. An., Vol. 15, pp. 723 (1984).

13. J. Harthong, Etudes sur la mécanique quantique (Astérisque, 1984).

14. J.M. Innocent and B. Torrésani, Wavelets and Binary Coalescence Detection, Applied and Computational Harmonic Analysis, Vol. 4, pp. 113-116 (1997).

15. C. Hory, N. Martin and A. Chehikian, Spectrogram segmentation by means of statistical features for non-stationary signal interpretation, preprint (2001), to appear in IEEE Trans. Signal Processing.

16. K. Kodera, R. Gendrin, and C. de Villedary, Analysis of time-varying signals with small BT values, IEEE Trans. ASSP Vol. 26, p. 64 (1978).

17. L.H. Koopmans, The spectral Analysis of Time Series. Probability and Mathematical Statistics, Vol. 22 (Academic Press, 1995).

18. J. Lamperti, Stochastic Processes (Springer-Verlag, 1977).

19. S. Mallat, A Wavelet Tour of Signal Processing, (Academic Press, 1998).

20. Y. Meyer, Oscillating patterns in image processing and in some nonlinear evolution equations, the Fifteenth Dean Jaqueline B. Lewis Memorial Lectures, Rutgers University (2001).

21. M. Morvidone, Détection temps-fréquence, application à la détection des ondes gravitationnelles $\mathrm{PhD}$ Thesis, Marseille (2002).

22. M. Morvidone, Time-scale chirp detection and estimation: Application to gravity waves detection II, in preparation.

23. M. Morvidone and B. Torrésani, Time-scale chirp detection and estimation: Application to gravity waves detection, Proceedings of the PSIP conference, Marseille, France pp. 264-269 (2001),

24. M. Morvidone and B. Torrésani, Time-scale approach for chirp detection, International Journal of Wavelet and Multiresolution Information Processing, Vol. 1, pp. 19-35 (2003).

25. B. Picinbono and W. Martin, Représentation des signaux par amplitude et phase instantanées, Annales des Télécommunications, Vol. 38 , pp. 179-190 (1983).

26. Ph. Tchamitchian and B. Torrésani, Ridge and Skeleton extraction from wavelet transform, in Wavelets and their Applications, M.B. Ruskai \& Al Eds, (Jones\&Bartlett, 1991).

27. K. Thorne, Gravitational waves, Proceedings of the Snowmass'94 Summer Study on Particle and Nuclear Astrophysics and Cosmology, eds. E. W. Kolb and R. Peccei, pp.398-425. (World Scientific, 1995).

28. H. Van-Trees, Detection, Estimation, and Modulation Theory. Part I, (John Wiley \& Sons, 1968). 